\title{
Traumatic spinal cord injuries in the rural region of Taiwan: an epidemiological study in Hualien county, 1986-1990
}

\author{
C Lan MD, ${ }^{1}$ JS Lai MD,${ }^{1} \mathrm{KH}$ Chang MD,${ }^{2}$ YC Jean PT, ${ }^{2}$ IN Lien $\mathrm{MD}^{1}$ \\ ${ }^{1}$ Department of Physical Medicine and Rehabilitation, National Taiwan University \\ Hospital No 1, Chang-teh Street, Taipei 10016, Taiwan; ${ }^{2}$ Department of Physical \\ Medicine and Rehabilitation, Buddhist Tz'u-Chi General Hospital, Taiwan, Republic of \\ China.
}

In order to survey the epidemiological characteristics of traumatic spinal cord injuries (SCI) in Hualien county, a retrospective study was conducted from January 1986 to December 1990 in four local general hospitals. During this period, 135 traumatic SCI patients were identified and 99 of them were residents of Hualien county. The estimated annual incidence of traumatic SCI in Hualien county was 56.1 per million population. As a result of injury 36 patients were tetraparetic, 33 tetraplegic, 12 paraparetic and 18 paraplegic. The male/female ratio was $4: 1$. The mean age of onset for male patients was 44 years, and that for female patients was 46 years. The major causes of SCI were traffic accident $(61.6 \%)$ followed by accidental falls $(23.3 \%)$. The average duration of hospitalisation ranged from 62 days in paraparetics to 132 days in tetraplegics. Ten patients died of respiratory failure or sepsis, and the mortality rate was $10.1 \%$. Our data revealed that traumatic SCI was prevalent in Hualien county in comparison with epidemiological studies elsewhere. The special population composition of Hualien county and the preponderance of the motorcycle as a transportation vehicle in this area might result in its unique epidemiological characteristics.

Keywords: spinal cord injury; epidemiology; incidence.

\section{Introduction}

Severe traumatic spinal cord injury (SCI) is a serious condition, resulting in severe disability or death. Patients who survive often have multiple complications. Most victims are young and in their most productive stage of life, causing not only them, but also their family members to suffer emotional and financial hardships. Consequently, a national registry is needed so that medical and social resources can be organised and integrated for their better management. At present there is still no national SCI registry in Taiwan; the only epidemiological study of SCI was reported by Chen and Lien. ${ }^{1}$ According to that report, the estimated annual incidence of traumatic SCI in Taipei city was 14.6 per million population. Taipei, located in northern Taiwan, is a densely populated city. Therefore, the reported data of SCI in
Taipei might be considered to be a profile in the urban area of Taiwan.

Kraus $^{2}$ has pointed out that the incidence rates of SCI in different studies were influenced by the research methods. In addition, we consider that the incidence of SCI is also determined by the sociological and demographic characteristics of the region studied. However, there are few studies which have discussed these factors. In order to elucidate the importance of sociological and demographic factors, we selected Hualien county for an epidemiological study of traumatic SCI. Hualien, located in eastern Taiwan, is surrounded by high mountains and faces the Pacific Ocean in the east. It is a geographically seclusive area and hence suitable for an epidemiological study. In 1988 , the population of Hualien county was about 354,000 and the population density 76 persons per $\mathrm{km}^{2}$. In contrast, Taipei has 
2.66 million inhabitants and the population density was nearly 10,000 persons per $\mathrm{km}^{2}$. In addition, the aborigines accounted for $23 \%$ of Hualien's population. Therefore, the socioeconomic, demographic, and geographic characteristics of these two regions are totally different.

This retrospective study was based on hospital records of patients with traumatic SCI who had been admitted to local hospitals in Hualien county from January 1986 to December 1990. Our purpose is to discover the incidence of traumatic SCI in Hualien county. In addition, the epidemiological characteristics of SCI in Hualien and Taipei will be compared, as being the best representative of the rural and urban regions in this island.

\section{Methods}

A retrospective review of hospital records of SCI patients was conducted from January 1986 to December 1990 in four general hospitals: the Buddhist Tz'u-Chi General Hospital, the Povincial Hualien Hospital, the Mennonite Christian General Hospital and the 805 Military Hospital. In Hualien, the above four have been classified as regional teaching hospitals, and only these hospitals have the capability to provide medical services for SCI patients. In addition, in our pilot study, we found that cases of SCI that occurred in Hualien were transferred to these hospitals for the acute care and/or further management. Records were obtained beginning from emergency service, and continuing through the intensive care phase and the rehabilitation stay. In order to ensure that no cases were missed, the records in the local Asociation of SCI Patients were also extensively surveyed. However, because of the limitations of a retrospective study, those who died before hospitalisation were not included in our study.

The definition of traumatic SCI was adopted from that proclaimed by Kraus et al as 'an acute, traumatic lesion of the spinal cord, including trauma to the nerve roots which resulted in varying degrees of motor and/or sensory deficit or paralysis'. ${ }^{3} \mathrm{~Pa}$ tients with nontraumatic SCI or patients with transient paralysis were excluded. Those who were not residents of Hualien were also excluded from the analysis.

A detailed chart review was done of all hospital records coded with the following rubric numbers of the International Classification of Disease (ICD): No. 806.0-9 fracture and fracture dislocation of vertebral column with spinal cord lesion; No. 968.0-9 spinal cord lesion without evidence of spine injury.

Case ascertainment included a follow up examination of the patients who were still alive during the time of study. This examination was performed by one of the authors, either at the outpatients department or during a home visit. Subjects were selected for the present study if they fulfilled the required criteria.

\section{Results}

\section{Incidence}

From January 1986 to December 1990, a total of 135 patients with a confirmed acute traumatic SCI were identified, 99 of whom were residents of Hualien county. During 1986-1990, the population of Hualien was about 353,000 , hence the average annual incidence of traumatic SCI in Hualien county was 56.1 per million population.

\section{Age and sex}

There were 79 male and 20 female SCI patients, the male/female ratio being $4: 1$. The average age at the time of injury was 44 years for males, and 46 years for females. Figure 1 illustrates the age distribution of SCI patients. The rate of occurrence for each age group was as follows: $20-29$ years $(19.2 \%) ; 30-39$ years $(19.2 \%) ; 40-49$ years $(15.1 \%) ; 50-59$ years $(15.1 \%) ; 60-69$ years $(17.2 \%)$. The age group below 20 and over 69 years accounted for $14.2 \%$ of all patients.

\section{Causes of SCI}

Table I lists the causes of SCI. Traffic accident was the leading cause, accounting for $61.6 \%$ of all subjects. Motorcycle collisions accounted for $74 \%$ of all traffic accidents. Falls accounted for $23.3 \%$ of all 


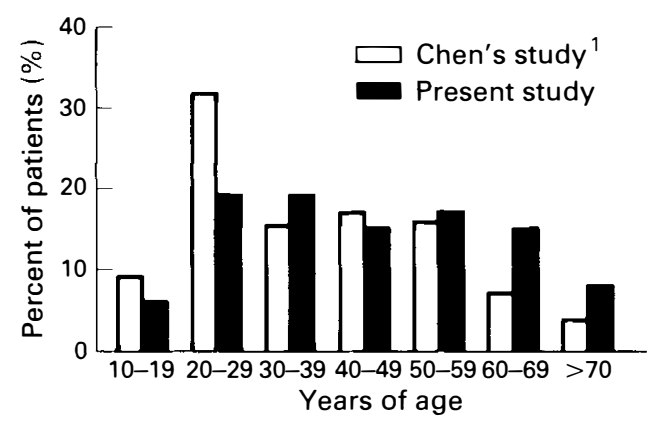

Figure 1 The age distribution of SCI patients. In Chen's report (1985), ${ }^{1}$ the peak occurrence rate of patients studied was in the 20-29 years group. In contrast, the present study showed almost the same occurrence rate in the age range $20-69$.

Table I Etiology of SCI

\begin{tabular}{lrc}
\hline Cause & \multicolumn{2}{c}{ Case No } \\
\hline Traffic accident & 61 & $(61.6 \%)$ \\
Accidental fall & 23 & $(23.3 \%)$ \\
Struck by object & 4 & $(4 \%)$ \\
Sports injury & 6 & $(6.1 \%)$ \\
Stab injury & 3 & $(3 \%)$ \\
Suicidal fall & 2 & $(2 \%)$ \\
\hline Total & 99 & $(100 \%)$ \\
\hline
\end{tabular}

subjects, and most occurred whilst quarrying, tunnelling or doing house construction. Other causes were sports injury, being struck by an object, stab injury and suicidal fall; these accounted for only $15.1 \%$ of all of the subjects.

\section{Functional impairment}

Table II shows the relationship between the cause of trauma and the resulting functional impairment. Tetraplegia and tetraparesis accounted for $69.7 \%$ of the subjects, while paraplegia and paraparesis accounted for the remaining $30.3 \%$. Eleven patients had nerve root injuries. Tetraparesis was the commonest neurological disability that was encountered. Complete paralysis was more frequent in thoracic cord injured patients, while incomplete paralysis was more frequent in cervical cord injured patients.

\section{Duration of hospitalisation}

The average hospital stay was 62 days for those who were paraparetic, 118 days for paraplegics, 102 days for tetraparetics and 132 days for tetraplegics.

\section{Causes of death}

Ten patients died of respiratory failure or from sepsis; all deaths occurred within one year after injury. Nine of the patients who died were tetraplegic and 6 of them died in the acute stage from respiratory failure. The remaining patient was paraplegic and died 8 months after injury from septicaemia resulting from multiple pressure sores. The overall mortaility rate was $10.1 \%$.

\section{Discussion}

According to the literature review of Kraus, ${ }^{2}$ the incidence of SCI ranged from

Table II Causes of SCI related to neurological deficits

\begin{tabular}{lllll}
\hline Cause & Paraplegia & Paraparesis & Tetraplegia & Tetraparesis \\
\hline Traffic accident & 8 & 2 & 24 & 27 \\
Accidental fall & 6 & 7 & 5 & 5 \\
Struck by object & 2 & 0 & 1 & 1 \\
Sports injury & 0 & 2 & 2 & 2 \\
Stab injury & 1 & 0 & 1 & 0 \\
Suicidal fall & 1 & 1 & 0 & $36(36.4 \%)$ \\
\hline Total & $18(18.2 \%)$ & $12(12.1 \%)$ & $33(33.3 \%)$ & \\
\hline
\end{tabular}


11.5 to 23 per million population in different regions. However, Kraus et $a l^{3}$ conducted a comprehensively designed study which surveyed traumatic SCI in 18 California counties during 1970-1971 and reported an incidence rate of 53.4 per million population. The incidence was very high because the victims who died before hospitalisation were included. Therefore, the authors stated that most of the reported incidence rates might be underestimated because victims who died before hospital admission were not usually included. In the present study, although the patients who died before hospitalisation were not included, the incidence of traumatic SCI in Hualien county was still astonishingly high in comparison with other reports, being 56.1 per million of the population: about fourfold the incidence reported in Taipei city.

There are several possible explanations for such a high incidence in Hualien county.

(1) Mass transportation is insufficient in Hualien county, and the motorcycle is the major transportation vehicle. According to the official data (1988), the number of registered motorcycles in Taipei was 261 per thousand of the population. However, the number in Hualien was 490 per thousand of the population. Motorcycle accidents surely expose the driver to an increased risk of a head injury and/or a cervical cord injury.

According to previous reports, traffic accidents are the most common cause of SCI. ${ }^{1,3,4-7}$ However, the types of motor vehicles should be taken into account. In Western countries, the major cause of traffic accidents is a car collision. In Southeast Asian countries, on the contrary, motorcycle accidents are the leading cause of trauma. According to an epidemiological study of head injury in Hualien, $82 \%$ of head injuries were related to a traffic accident, and $71 \%$ of the traffic accidents involved motorcycle(s). ${ }^{8}$ In the present study, traffic accidents accounted for $62 \%$ of all SCI cases, and $74 \%$ of the traffic accidents were motorcycle related. Consequently, it is not surprising that the cervical SCI patients accounted for $70 \%$ of all of the casualties.

(2) Hualien is a mountainous area, and quarrying and mining are the main industries, hence falls are prevalent. In the present study, falls accounted for $23.3 \%$ of all of the patients, and $57 \%$ of the falls resulted in paraplegia or paraparesis.

(3) Alcoholism also plays an important role in the high incidence of traffic accidents in Hualien county. In Taiwan, the prevalence of alcoholism was higher in the rural regions than in the urban regions. Hwu et $a l^{9}$ reported that the lifetime prevalence of alcohol abuse in Taiwan Chinese was $3.4 \%, 8.0 \%$, and $6.3 \%$ in the metropolitan city, small town, and village respectively. In addition, alcoholism is prevalent amongst the Taiwan aborigines, resulting in serious sociomedical problems. The reported lifetime prevalence of alcohol abuse was $11.6 \%$ in the Atayal tribe, and $14.2 \%$ in the Paiwan tribe, which are the two major ethnic groups of Taiwan aborigines. ${ }^{10}$ Alcohol may delay response time, hence a drunk driver is more likely to be involved in a motor vehicle collision. Hung et $\mathrm{al}^{8}$ reported that at least $26 \%$ of the head injuries that occurred in Hualien might be related to alcohol intake. In the present study, we found that at least $21 \%$ of the SCI patients had a significant alcohol intake before the accident occurred.

The male/female ratio of traumatic SCI in Hualien county was $4: 1$. Chen et al ${ }^{1}$ reported the ratio was 4.9:1 in Taipei city. Kuhn et $\mathrm{al}^{4}$ have pointed out that the male/female ratio might reflect socioeconomic and cultural differences.

The peak incidence of traumatic SCI, as is reported in many studies, is usually within the age range of 20 to 29 years. ${ }^{1.5,6,11-13}$ Because people of this age are involved in many significant activities, a higher risk of trauma may be expected. Chen et al ${ }^{1}$ reported that $31.8 \%$ of traumatic SCI patients were in the 20-29 years age group. In the present study, patients of this age group comprised only $19.2 \%$ of all cases (Fig 1 ). 
According to these data, the percentage of young SCI patients in Hualien appeared to be lower than that of Taipei city. Therefore, we speculated that the population composition of Hualien and Taipei was different. According to the official data: people of $20-29$ years of age accounted for $19.5 \%$ of the total population in Hualien, while the same age group accounted for $17.9 \%$ in Taipei. At first glance, the proportion of young people in Hualien did not appear to be lower than that of Taipei. However, as we explored further, we found that the official data might not represent the actual population composition. Hualien is basically an agricultural area, and people who live there are usually underemployed. Therefore many of them, most being young, leave their home to look for jobs. However, many people who work elsewhere still retain their residency as being registered in Hualien. Consequently, the 'occult' population composition may be different from that of the official data. Although the actual number of people who worked outside Hualien was difficult to determine, it has been estimated unofficially that the number was not less than 30,000. Therefore, although the incidence of traumatic SCI appeared to be low in the 20-29 years age group, it did not represent the actual risk to this age group; rather it reflected the unique demographic and sociological characteristics of Hualien county.

Chen et al $^{1}$ reported that the mean onset age of SCI in male patients in Taipei city was 36.3 years, and that of female patients was 33.7 years. In the present study, the mean onset age of SCI patients in Hualien county was 44 years in males, and 46 years in female patients. The mean onset age of SCI patients in Hualien county was about 10 years older than that of Taipei city. As we have explained earlier, part of the difference may be attributed to the unequal population compositions.

The duration of hospitalisation in the present study ranged from 62 days for paraparetics to 132 days for tetraplegics. In Taipei city, Chen et al ${ }^{1}$ reported that the duration ranged from 44 days for paraparetics to 100 days for tetraplegics. BieringSørensen et $a l^{7}$ pointed out that the data from Chen's report revealed very short admissions, and he questioned if the complete rehabilitation period was included. In fact, the duration of hospitalisation is difficult to compare because the social welfare system and the comprehensiveness of medical services are very diverse in different regions.

Ten patients died of respiratory failure or sepsis; the overall mortality rate was $10.1 \%$. This was higher than that of the report of Chen for Taipei city (6\%). However, the difference of mortality might partly be attributed to the high incidence of cervical $\mathrm{SCI}$ in Hualien. Because the actual number of patients who died before hospitalisation was difficult to determine, we expect that the real mortality might be much higher than the above mentioned ratio.

In summary, our data revealed that traumatic SCI was prevalent in Hualien county and the incidence rate was very high in comparison with other reports. In this region, the incidence of cervical cord injury was also surprisingly high. From the sociological point of view, the result could be explained by the following facts. (1) Motorcycles were widely used as a means of transport. Although motorcycles are cheap and convenient, the risk of a serious SCI occuring in an accident was high. (2) Mining and quarrying are the main industry in Hualien, hence the risk of injury from a fall was high. (3) The prevalence of alcohol abuse is relatively high in the rural area of Taiwan, especially amongst the aborigines. A drunken person is more likely to be involved in a serious trauma. The lower incidence of SCI in the 20-29 age group might be attributed to the unique demographic characteristics of Hualien county. Therefore, we conclude that the sociological and demographic characteristics are important, and they must be taken into account in an epidemiological study of SCI.

\section{Acknowledgement}

The authors gratefully acknowledge the collaboration of Dr LY Chu (Department of Physical Medicine and Rehabilitation, Provincial Hualien Hospital) in this study. 


\section{References}

1 Chen CF, Lien IN (1985) Spinal cord injuries in Taipei, Taiwan 1978-81. Paraplegia 23: 364-370.

2 Kraus JF (1980) Injury to the head and spinal cord. The epidemiological relevance of the medical literature published from 1960-1978. J Neurosurg 58: S3-S10.

3 Kraus JF, Franti CE, Riggins RS, Richards D, Borhani NO (1975) Incidence of traumatic spinal cord lesions. J Chron Dis 28: 471-492.

4 Kühn W, Zäch GA, Köchlin PH, Urwyler A (1983) Comparison of spinal cord injuries in females and in males, 1979-81 Basle. Paraplegia 21: 154-160.

5 Bracken MB, Freeman DH Jr, Hellenbrand K (1981) Incidence of traumatic hospitalized in spinal cord injury in the United States 1970-1977. Am J Epidemiol 113: 615-622.

6 Fine PR, Kuhlemeier KV, Devivo MJ, Stover SL (1980) Spinal cord injury: an epidemiological perspective. Paraplegia 17: 237-250.

7 Biering-Sørensen F, Pedersen V, Clausen S (1990) Epidemiology of spinal cord lesions in Denmark. Paraplegia 28: 105-118.

8 Hung CC, Chiu WT, Tsai JC, Laporte RE, Shih CJ (1991) An epidemiological study of head injury in Hualien county, Taiwan. J Formosan Med Assoc 90: 1227-1233.

9 Hwu HG, Yeh EK, Yeh YL, Chang LY (1988) Alcoholism by Chinese Diagnostic Interview Schedule: A prevalence and validity study. Acta Psychiatr Scand 77: 7-13.

10 Hwu HG, Yeh YL, Wang JD, Yeh EK (1990) Alcoholism among Taiwan aborigines defines by the Chinese Diagnostic Interview Schedule: A comparison with alcoholism among Chinese. Acta Psychiatr Scand 82: 374-380.

11 Gjone R, Nordlie L (1979) Incidence of traumatic paraplegia and tetraplegia in Norway: a statistical survey of the years 1974 and 1975. Paraplegia 16: 88-93.

12 Minaire P, Castanier M, Girard R, Beard E, Deidier C, Bourret J (1979) Epidemiology of spinal cord injury in the Rhône-Alpes region, France, 1970-75. Paraplegia 16: 76-87.

13 Stover SL, Fine PR (1987) The epidemiology and economics of spinal cord injury. Paraplegia 25: $225-228$. 